\title{
SCHULDENBREMSE
}

\section{Solidarität bedeutet} Umverteilung

\author{
VON ULRICH SCHNEIDER UND \\ CHRISTIAN WOLTERING \\ Dr. Ulrich Schneider ist Haupt- \\ geschäftsführer und Christian \\ Woltering ist Referent für fach- \\ politische Grundsatzfragen des \\ Paritätischen Wohlfahrtsverban- \\ des-Gesamtverband e. V. \\ Internet
}

www.der-paritaetische.de

\author{
Ende letzten Jahres hat sich der Verbandsrat des \\ Paritätischen Wohlfahrtsverbandes vor dem Hintergrund \\ der Schuldenbremse für eine rigorose Kurkorrektur in der \\ Finanzpolitik hin zu einer solidarischen Finanzierung des \\ Sozialstaates ausgesprochen. Der Wohlfahrtsverband \\ hat damit erstmals seine Zurückhaltung in \\ finanzpolitischen Angelegenheiten aufgegeben.
}

Auf fast jeden Haushaltsengpass, jede Rezession und jede Finanz- oder Staatskrise wurde in der Vergangenheit mit der Aufnahme neuer Schulden reagiert. Ergebnis: Die Bundesrepublik Deutschland stand zum Jahresende 2011 mit rund 2,03 Billionen Euro bei seinen Gläubigern in der Kreide. Der Schuldenberg beträgt inzwischen über 80 Prozent des Bruttoinlandsproduktes, also über vier Fünftel des Gesamtwerts aller Waren und Dienstleistungen, die innerhalb eines Jahres in Deutschland produziert wurden. Unzweifelhaft und unbestritten sind das besorgniserregende Tendenzen.

Handelt es sich also um eine ausweglose Situation, ist die Merkelsche Austeritätspolitik Schuldenbremse gar »alternativlos«? Mitnichten. Den Kassandrarufen derjenigen zum Trotz, die den Haushalt mittels Kahlschlag sanieren wollen, gibt es nämlich auch gute Nachrichten. Stellt man dem Verschuldungsbetrag das Geldvermögen der privaten Haushalte in Deutschland gegenüber, so relativiert sich die Summe beträchtlich. Laut Berechnungen der Bundesbank betrug das Geldvermögen der Deutschen im Jahre 2011 gut 4,7 Billionen Euro - wobei diese Zahl deutlich schneller wächst, als die öffentliche Verschuldung.

Privates Geldvermögen mag im Einzelfall sehr angenehm sein. Das Problem ist, dass es ungeheuer ungleich innerhalb der Gesellschaft verteilt ist. Im Schnitt verfügte eine Person über 17 Jahren im Jahr 2007 laut des Deutschen Instituts für Wirtschaftsforschung über ein individuelles Nettogesamtvermögen von rund 88.000 Euro. Dass dies jedoch auf den allergrößten Teil der Bevölkerung nicht zutrifft, dürfte offensichtlich sein. Durchschnittswerte sind daher von begrenzter Aussagekraft. Teilt man aber die Gesellschaft in zehn Einkommensklassen ein, in sogenannte Dezile, so zeigt sich, dass die drei obersten Dezile im Jahr 2007 über 91 Prozent des Gesamtvermögens unserer Gesellschaft auf sich vereinen. Dem Rest der Bevölkerung bleiben knappe neun Prozent.

Zusammengefasst sind also drei Faktoren bedeutsam: ersten eine wirklich immense öffentliche Verschuldung von rund zwei Billionen Euro, zweitens fast fünf Billionen Euro privates Geldvermögen, drittens eine höchst ungleiche Verteilung des Vermögens auf die Bevölkerung. Diese ist ein deutlicher Ausdruck dafür, dass im Sozialgefüge unseres Landes etwas nicht stimmen kann. Dass eine solch einseitige Konzentration der Vermögensverhältnisse dazu beitragen kann, ein demokratisches System in eine reale Krise zu stürzen, zeigte sich in den Protesten der kürzeren Vergangenheit in Ländern wie England, Griechenland oder auch in Frankreich.

Vor dem Hintergrund des Schulden- 
bergs in unserem Land und der Feststellung, dass man allein durch Zurückhaltung der Ausgaben diese nicht überwinden wird, ohne bleibende Flurschäden im Sozialgefüge unserer Gesellschaft zu hinterlassen, müssen wir uns mit Alternativen befassen. Welche Steuerarten haben aber Potenzial für sachgerechte Mehreinnahmen?

Hier sei zunächst die Erbschaftssteuer genannt. Nach Studien des Deutschen Instituts für Altersvorsorge werden in den nächsten zehn Jahren 2,6 Billionen Euro vererbt. Diese gigantische Summe wird jedoch ebenfalls extrem ungleich über die Gesellschaft verteilt. Die Staatseinnahmen sind zum jetzigen Zeitpunkt mit rund fünf Milliarden Euro eher marginal. Da der Spitzensatz der Erbschaftssteuer für enge Verwandte unter dem Höchstsatz der Einkommenssteuer liegt, reduziert die Erbschaftssteuer auch noch die Progressivität des Steuersystems. Hätten wir ein reales Erbschaftssteueraufkommen von wenigstens zehn Prozent, dann hätten wir in den nächsten zehn Jahren Finanzmittel im Wert von 260 Milliarden Euro mehr im Haushalt.

Der zweite Punkt betrifft die Vermögenssteuer. Diese existierte in Deutschland auf geringem Niveau bis zum Jahr 1997 und wurde dann von der Regierung ausgesetzt. Grund für die Aussetzung der Steuer war ein Urteil des Bundesverfassungsgerichtes im Jahr 1997, das die Besserstellung von Immobilien im Vergleich zu anderen Vermögen für verfassungswidrig erklärte. Doch anstatt Immobilien höher zu bewerten beziehungsweise deren Wert neu bestimmen zu lassen, entschied sich die damalige Bundesregierung für eine Aussetzung der Vermögensteuer. Will man also die beschriebenen Verteilungsungerechtigkeiten korrigieren und das Vermögen dem Wohle der Allgemeinheit zukommen lassen, so bleibt einem kaum eine andere Wahl als direkt das Substanzvermögen der Reichen und Superreichen in unserem Staat zu besteuern. Selbst bei geringer prozentualer Besteuerung einhergehend mit hohen Freibeträgen, würde die Steuer Mehreinnahmen in Höhe von zehn Milliarden Euro einbringen.

Zu guter Letzt sei die Finanztransaktionssteuer noch genannt, deren Effekt bei einer Minibesteuerung von 0,01 Prozent ebenfalls bei Mehreinnahmen von über zehn Milliarden Euro liegen.

\section{Fazit}

Allein die drei hier genannten Steuerarten würden so viele Mehreinnahmen bringen, dass die negativen Effekte einer Schuldenbremse ausgeglichen werden könnten. Dabei geht es nicht mal nur um die Umverteilung hin zu sozial Schwachen. Der Großteil unserer Gesellschaft würde durch Kürzungen im Bereich der sozialen Sicherungssysteme, der öffentlichen Infrastruktur und der allgemeinen Versorgung zu leiden haben.

Kompensieren könnten diese Einschnitte nur diejenigen, die genug Vermögen haben, um auf die öffentliche Infrastruktur in weiten Teilen nicht angewiesen zu sein. Also der geringe Prozentsatz unserer Mitbürger, der seine Kinder nicht ins städtische Schwimmbad schicken muss, weder auf staatliche Schulen noch auf allgemeine Gesundheitsversorgung angewiesen ist, und sich Kunst lieber ins eigene Wohnzimmer hängt, anstatt sie sich im Museum anzuschauen. Der ganz große Rest wird das grundgesetzlich verankerte Spargebot, und den daraus resultierenden Verteilungskampf am eigenen Leibe miterleben.

Daher muss das Plädoyer lauten, sich entweder wieder stärker für Umverteilung in unserer Gesellschaft einzusetzen, oder sehenden Auges die soziale Spaltung in unserem Land zuzulassen. Der Schuldenbremse als sozialpolitischem Spaltpilz, kann und muss entgegengewirkt werden. Vor allem aber hätte man die Mittel, wieder ein Stück weit mehr gesellschaftliche Vermögensgerechtigkeit zu schaffen. Die Oppositionsparteien haben sich in der Vergangenheit schon mehr oder weniger vehement für Umverteilung eingesetzt. Daran werden sie sich im Falle eines Wahlsieges von uns messen lassen müssen.

Eine ausführliche Begründung der finanzpolitischen Forderungen des Paritätischen Woblfabrtsverbandes mit Quellenhinweisen findet sich auf der Website des Verbandes (bttp://www. der-paritaetische.de/startseitelartikel/ news/wir-haben-noch-nie-geteilt-obneeine-zielgerichtete-umverteilungspolitik-droht-unsere-gesellschaf).

\section{Lohnnebenkosten Gesundheit}

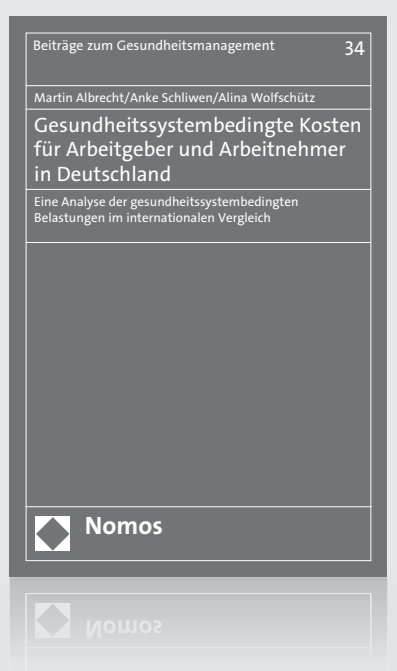

Gesundheitssystembedingte Kosten für Arbeitgeber und Arbeitnehmer in Deutschland

Eine Analyse der gesundheitssystembedingten Belastungen im internationalen Vergleich

Von Martin Albrecht, Anke Schliwen und Alina Wolfschütz

2012, 139 S., brosch., 18,-€ ISBN 978-3-8329-7249-3

(Beiträge zum Gesundheitsmanagement, $B d$. 34)

Die Belastung der Arbeitgeber durch Lohnzusatzkosten wird in Deutschland seit Jahren als gravierendes wirtschafts- bzw. arbeitsmarktpolitisches Problem diskutiert. Die vorliegende Studie ermittelt die Höhe der Belastung der Arbeitgeber durch gesundheitssystembedingte Kosten und vergleicht sie zwischen Branchen sowie international.

www.nomos-shop.de/14403

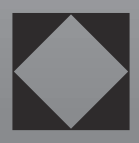

Nomos 